\title{
LENGUA ORIGINAL Y TRADUCCIÓN CASTELLANA EN CANDIDO DE LEONARDO SCIASCIA
}

SALVATORE BARTOLOTTA

UNED. Madrid

\section{RESUMEN}

En el presente artículo, se analiza Candido de Leonardo Sciascia partiendo, linguísticamente, de la revisión del concepto de dialectalidad, que el autor asume conscientemente, más de forma intuitiva que académica. Se revisa la dialectalidad, entendida en su variedades diatópicas, diafásicas y diastráticas y también en contraste con el resto de sus obras. Dicho análisis anticipa la posterior forma de articulación, tanto de los diferentes niveles del texto original, como de su correspondiente traducción al castellano; donde se constatarán enormes dificultades de adaptación por la presencia constante de numerosas formas dialectales.

\section{INTRODUCCIÓN}

Es por la fluidez y ligereza que caracterizan al conte philosophique ${ }^{1}$, por lo que Leonardo Sciascia lo elige como modelo para crear su relato, Candido ovvero un sogno

«Es la brevedad, decimos, de la contundencia, la nota dominante en los géneros y recursos volterianos. Un estilo breve conformado por frases como latigazos, de sonido seco y fuerte, aun al precio de simplificar argumentos e ideas. [...] Pero, sobre todo, brevedad como expresión de un discurso que tiende a pasar a grandes zancadas sobre los grandes temas [Ramoneda, 1996: 376]". 
fatto in Sicilia ${ }^{2}$, una de sus obras más notables. Publicado en Turín por Einaudi en 1977, la breve novela brota de la desilusión de Sciascia ante su fracasada experiencia de consejero comunista en el ayuntamiento de Palermo, y se presenta surcada por-un profundo y consciente autobiografismo. Es una especie de recreación del Candide de Voltaire, como anota el mismo Sciascia [1977: 139] citando a Montesquieu.

Dice Montesquieu che «un'opera originale ne fa quasi sempre nascere cinque o seicento altre, queste servendosi della prima all 'incirca come i geometri si servono delle loro formule». Non so se il Candide ${ }^{[3]}$ sia servito da formula a cinque o seicento altri libri. Credo di no, purtroppo: ché ci saremmo annoiati di meno, su tanta letteratura. Comunque, che questo mio racconto sia il primo o il seicentesimo, di quella formula ho tentato di servirmi. Ma mi pare di non avercela fatta, e che questo libro somigli agli altri miei. Quella velocità e leggerezza non è più possibile ritrovarle: neppure da me, che credo di non avere mai annoiato il lettore. Se non il risultato, valga dunque l'intenzione: ho cercato di essere veloce, di essere leggero. Ma greve è il nostro tempo, assai greve.

En los breves capítulos, introducidos por largos y complejos títulos, se cuentan las desventuras de un joven siciliano de buena familia, Candido Munafò, nacido en 1943 en una gruta de la isla, precisamente durante la noche del desembarco anglo-americano, cuyas desventuras concluyen en 1977, cuando encontrándose en París revive el mito del mayo de 1968.

También aquí, como en el célebre modelo volteriano ${ }^{4}$, el tema central está representado por la insuficiencia de los esquemas interpretativos acuñados en el pasado por los filósofos y actualmente por los políticos; la mistificación de fórmulas y dogmas elaborados por intelectuales que se denominan progresistas. La paradoja, que recorría toda la obra de Voltaire ${ }^{5}$, está presente también en la obra de Sciascia, porque el siciliano arrastra sus mismas contradicciones. En este juego de paradojas - que no por casualidad halló en el cuento su más feliz expresión - existe una razón caprichosa y vigorosa y una actividad incesante, que representa muy bien la plenitud de fuerzas de la joven sociedad moderna y burguesa.

Según Candido: «las cosas siempre son sencillas». Es la ansiedad por llegar a su corazón, llamarlas por su propio nombre, lo que le provoca tantos líos. Ignorado, o mejor abandonado, por el padre y por la madre, y confiado al cuidado de su abuelo, general de la milicia fascista y más tarde diputado democristiano, Candido aprende pronto a pensar

\footnotetext{
${ }^{2}$ Véase Sciascia (1977) y Ambroise (1989: 347-462). Numerosos son los escritos críticos sobre el Candido de Sciascia, entre ellos recuérdese Siciliano (1977). Baldi (1978: 161-68), Luperini (1978: 105-07), Cottone (1980: 128-31), Chevello (1989: 39-46), Deidier (1993: 35-68), Scarano (1993: 297-316), Ambroise (1996: 130-36), Bernardini Napoletano (1996: 65-74), D’Anna (1996: 75-84), Santangelo (1996: 85-120), Bandinelli (1998: 187-96) y Monforte (2001: 197-201).

${ }^{3}$ La novela homónima de Voltaire, Candide fue publicada en 1759.

${ }_{4}^{4}$ "Voltaire es uno de los pensadores que han influidos en las ideas, en las costumbres y en la civilización, erigiéndose en referencia de estilo y actitudes, lo que rebasa el valor de su obra intelectual. Es el principal punto de referencia de la figura moderna del intelectual como conciencia crítica de la sociedad [Ramoneda, 1996: 375]».

${ }^{5}$ Sobre el concepto de paradoja en Voltaire véase Ramoneda (1996: 380-85).
} 
por sí mismo. Empieza a enfrentarse con la hipocresía que rige la ficción de la llamada "vida civil». El «pequeño monstruo» terminará en manos de un preceptor arcipreste y encontrará en él otro hereje por vocación, un interlocutor ideal que lo encamina hacia el Partido Comunista. Juntos se enfrentarán con las dos grandes iglesias de nuestro tiempo.

Una vez más, en ese brillante apólogo, entrelazado por el hilo de la ironía sardónica, habitual para sus lectores, Sciascia no abandona las invenciones narrativas y las incursiones polémicas: las ambiguiedades y las contradicciones del mundo de hoy, el comunismo, un cierto modo de vivir y entender el catolicismo, el confluir del catolicismo en el comunismo y del comunismo en el catolicismo, la incapacidad de ambos para asumir y actuar según los ideales que proponen teóricamente.

Más adelante, Candido se alejará del Partido por culpa de la hipocresía de los «compañeros», que miran con malos ojos su relación con una joven criada. Por ello decide dejar definitivamente su pueblo en compañía de una prima con la que acaba en París donde, inesperadamente, conocerá a sus padres. Candido más que una novela es una velada autobiografía: el protagonista, que desde su Sicilia natal termina en París, revive las dramáticas tensiones y desilusiones políticas del mismo Sciascia, eligiendo por fin la capital francesa como único lugar donde todavía es posible sentir y hacer sentir la voz de la razón.

\section{LA LENGUA Y LA DIALECTALIDAD}

Antes de entrar en consideraciones generales sobre la lengua y la dialectalidad ${ }^{6}$ de la obra objeto de análisis, parece conveniente dar cuenta del juicio que el propio Sciascia concede a su escritura. Es un autor preocupado por el lenguaje y sus variantes dialectales y léxicas y así lo hace constar en diversas entrevistas.

En la entrevista con Marcelle Padovani ${ }^{7}$, a la pregunta de si

la sua esperienza ha implicato una rottura con la Sicilia in quanto dialetto, esperienza letteraria e modo di esprimersi,

Leonardo Sciascia responde:

Quando mi accade di rileggere un brano dei miei libri, mi accorgo che la mia sintassi si è fatta progressivamente meno dialettale, che oggi mi si è fatto più raro l'uso di 'sicilianismi', che le Parrocchie è zeppo di dialetto mentre Todo modo ne è esente, ma anche che l'insieme del processo è andato avanti del tutto naturalmente.

En otra entrevista concedida a Walter Della Monica $^{8}$, a la pregunta de si el dialecto había «inciso sulla qualità linguistica della [sua] opera», Sciascia reconfirma su juicio:

\footnotetext{
${ }^{6}$ Para los estudios sobre lengua y dialectalidad en Sciascia véase Sgroi (1990a: 177-412), Vecchio (1990: 49-57), (1991: 83-94) y (1998: 173-86), Coletti (1993: 342-45 y 375-79) y Bucca (1999: 141-50).

${ }^{7}$ Cfr. Padovani (1979: 77).

${ }^{8}$ Cfr. Della Monica (1981: 41).
} 
Conosco benissimo il siciliano [...] lo parlo abitualmente: in Sicilia e quando mi ritrovo tra siciliani. E credo abbia avuto parte importante nelle prime cose che ho scritto. Da un libro all'altro, il mio scrivere è diventato sempre più italiano: un fatto naturale, assolutamente involontario.

Desde el punto de vista linguístico, es ante todo oportuno decir que la dialectalidad de Leonardo Sciascia no debe considerarse como un uso del dialecto siciliano (o de una variedad diatópica) en contraposición con el código literario italiano, como si nos encontráramos ante un escritor dialectógrafo o bilinguie, dialectógrafo e italógrafo, como es el caso de otros autores de la literatura italiana no sólo contemporánea.

La dialectalidad de Sciascia no es ni siquiera un ejemplo de code-switching, de conmutación, es decir, de códigos distintos que van desde el dialecto siciliano hasta el italiano literario, observable en el interior de un mismo texto a modo de alternancia de unidades lingüísticas meramente dialectales en un contexto italiano, a la manera, por ejemplo, de $\mathrm{Gadda}^{9}$. El code-switching está ausente, salvo en rarísimas excepciones, en la producción de Sciascia; y, como se verá, es completamente inoperante en Candido.

La dialectalidad de Sciascia está considerada, en general, como un ejemplo de variedad diatópica del código escrito italiano, que resulta de la interferencia de la lengua literaria con el dialecto (hablado) siciliano y de algunos restos de la variedad agrigentina (el autor, como se sabe, es natural de Racalmuto, un pueblo de la provincia de Agrigento). El resultado de la interferencia se puede considerar tanto negativo como positivo. En el primer caso (interferencia negativa) la forma lingüística de origen dialectal es específica en la prosa regional siciliana. En el segundo caso (interferencia positiva) la forma linguiística (a) aunque derive del dialecto, se ha difundido más o menos ampliamente en otras variedades escritas del italiano en las que antes estaba ausente y, sincrónicamente, tiende, por lo tanto, a perder todas las connotaciones regionales; o (b) aunque sea suprarregional en la medida en que está presente en otras variedades del italiano, es paralela a la dialectal y, por lo tanto, se ve frecuentemente reforzada. En el caso de la interferencia positiva, es condición primera y sine qua non que el lector conozca el dialecto para poder realizar una correcta localización de las formas mimetizadas, de alta frecuencia, pero poseedoras de un notable substrato dialectal.

Sin embargo, la misma dialectalidad diatópica, en Candido, no carece - como se verá - de reflejos idiolectales, estrictamente estilísticos e individuales. Ello significa que la interferencia siciliano / italiano aparece cuidadosamente filtrada por el autor, que reelabora el estímulo dialectal inicial. De este modo se convierte en un hecho más difícil de localizar, sobre todo para los no sicilianófonos, dada la perfecta simbiosis entre los rasgos del código literario italiano y los del código oral siciliano.

\footnotetext{
${ }^{9}$ Carlo Emilio Gadda procede violentemente contra al lenguaje, fuerza los equilibrios normales, se aparta de la armonía clásica, mezcla múltiples niveles lingüísticos y estilísticos, variados dialectos italianos, heterogéneas fórmulas de la lengua literaria, fragmentos de lenguas extranjeras, jergas y lenguas especiales y técnicas del uso corriente. Se trata de una gama vastísima que va de la lengua más popular a la más culta y arcaizante, manierista y barroca, jocosamente artificial, erudita y pedantesca. Sobre el expresionismo, el plurilingüismo y el pastiche lingüístico de Gadda cfr. Ferroni (1991: 319-20).
} 
Junto a esta característica diatópica general, la dialectalidad de Sciascia presenta el rasgo específico de una dialectalidad diafásica; es decir, de una variedad funcional-contextual. No solamente por el uso de subcódigos especiales, bastante exiguos (algunos tecnicismos) en Candido, sino también por el recurso a registros y niveles medios y coloquiales, calcados del dialecto (hablado). En las partes descriptivo-narrativas se alternan registros y niveles, lógicamente, más formales y cultos en función de los diversos componentes del hecho lingüístico. Todo ello contribuye a crear una prosa ágil y veloz, tan del gusto del autor, particularmente visible en Candido.

La dialectalidad diafásica de Candido aflora en las partes narrativas y descriptivas; es decir, en el nivel más formal del escritor que narra - como sugiere Pellegrini (1970: 368) - en tercera persona, así como en las partes dialogadas de los personajes, o lo que es igual, en el nivel menos elaborado. Aquélla, por lo tanto, no sólo caracteriza la lengua de los más variopintos personajes, sino también la del propio escritor, que en calidad de narrador se inmiscuye en la obra (en tercera persona y a veces en primera del plural) y, a menudo, utiliza el estilo indirecto libre, confundiéndose con los mismos personajes.

La dialectalidad, además de aflorar como marca geográfica y funcional, se presenta a un tiempo, como variedad diastrática (sociolectal), para indicar el status socio-económico y la posición de clase de los protagonistas. Esto es comparable, por ejemplo, con el uso de palabras malsonantes e improperios. Rasgo, éste, mucho más marcado en otros textos: por ejemplo (a) la utilización del léxico jergal en $I$ mafiosi ${ }^{10}$, cuya función críptica es paradójicamente anulada en el propio texto, a través de las continuas intervenciones metalingüísticas de los personajes ${ }^{11}$; (b) el uso del sículo-americano por parte de los protagonistas de La zia d'America ${ }^{12}$, cuyos términos están sutilmente glosados en las citas por el autor ${ }^{13}$. Pero varios rasgos diastráticos de la prosa de Candido se alejan considerablemente de lo específico de una región y son por lo tanto panregionales.

Así, la dialectalidad, filtrada y reflejada en la lengua del escritor siciliano, presenta aspectos diversos, distribuidos y jerarquizados de modo distinto en los planos del relato: diatópicos (interferencia negativa y positiva del italiano literario en el dialecto siciliano), diafásicos (registro medio-informal y subcódigos), diastráticos (subcódigos, jergas) e idiolectales (estilísticos) ${ }^{14}$.

\section{LA LENGUA DE LA VERSIÓN ESPAÑOLA}

Se puede concretar que estas variantes fundamentales explícitas e implícitas del texto original (diastráticas, diatópicas, diafásicas e idolectales) convergen en la traduc-

10 Véase Sciascia (1966) y (1976) y Ambroise (1991: 1229-96).

"Cfr. Sgroi (1987: \$2.4).

${ }^{12}$ Cuento incluido en Sciascia (1958). Véase también Ambroise (1987: 173-222).

${ }^{13}$ Véase Sgroi (1984: 411-45).

${ }^{14}$ Se definen y aclaran todos estos aspectos de acuerdo con las múltiples manifestaciones recogidas en los diferentes niveles de análisis (fonológico, morfo-fonológico, morfosintáctico y léxico) siguiendo las propuestas del entramado lingüístico de Weinreich (1953), (19632) y (tr. it., 1974), que estudia los mecanismos de la interferencia en los distintos planos del lenguaje, adaptadas posteriormente por Sgroi (1990a: 177-412) y (1990b: 367-432) para el análisis de textos italianos. Por otra parte, se siguen las descripciones estructurales y geolingüísticas del italiano y de los dialectos italianos proporcionadas por Rohlfs (1949-54) y (tr. it., 196669) y Tekavčic (1962-67) y (tr. it., 1980). 
ción y producen un efecto de choque que el traductor tiene que adecuar. Las elecciones realizadas por el traductor, tanto gramaticales como léxicas, configuran una nueva obra cuyo resultado final puede asemejarse al original o alejarse mucho de él. El problema teórico que entraña cualquier texto de mayor o menor complejidad formal y estilística, o la dificultad conceptual de los contenidos, se hace todavía más complejo en esta obra por la presencia constante de numerosas formas dialectales. Dichas variantes reflejan una modalidad de lengua que trasluce una determinada forma mentis, lo cual entraña una gran dificultad de traducción, sin que ello signifique que sea imposible. La traducción no puede limitarse a una labor de recodificación, sino que debe constituirse en un proceso de recreación del universo sociocultural y estilístico de la obra, difícil de transmitir en una lengua distinta a la del original.

Por lo que respecta al plano de la traducción ${ }^{15}$, en la edición analizada ${ }^{16}$, en primer lugar se echa de menos una nota del traductor que justifique sus opciones y los criterios sobre los que se fundamentan las mismas. El mayor error en que la traductora ha incumido se acerca a lo que Mounin (1965: 139) llama la disparidad, es decir, la falta de unidad lingüística en el texto traducido. La versión española que se aleja ostensiblemente del original, oscila entre matices regionales y cultos, mientras que el original conserva un nivel medio informal. Las dificultades de la traducción se resuelven sin criterios bien definidos y sin tener en cuenta el registro usado por el original. La regla de la unidad de la lengua y el rechazo de las disparidades estilísticas imponen la elección de un registro de traducción, al que se debe permanecer fiel.

De forma general, en la traducción se observan determinados rasgos que se pueden sintetizar en lo siguiente: la dificultad de traducir el registro dialectal, la falta de unidad en el criterio que debe primar en la selección entre las opciones posibles y la heterogeneidad en los grados de aproximación al original optando por la hispanización o la italianización.

En cuanto a la dificultad de traducir el registro dialectal se pone en evidencia el caso del uso de hermandad de la mafia / omertà que pretende acercar el texto al lector hispanizándolo a costa de la pérdida de su fuerza expresiva. Una posible solución hubiera

${ }^{15} \mathrm{Y}$ en nuestro caso, «por lo que respecta a sus lectores españoles, - según Navarro Salazar (1999) generalmente Sciascia está considerado como el escritor de la mafía y también como el escritor que busca la verdad y la justicia. La mayoría de sus novelas han sido traducidas al castellano y a otras lenguas peninsulares, pero obras enormemente significativas para comprender su trayectoria de escritor comprometido como son La corda pazza, Pirandello e la Sicili, Pirandello e il Pirandellismo, y Occhio di capra siguen esperando. La causa de tal dicotomía habría que buscarla en el hecho de que el lector medio español no puede captar la concatenación de implicaciones históricas y políticas que fundamentan este tipo de obras de ensayo".

${ }^{16}$ Para el análisis de la lengua de la versión española, Cándido o un sueño siciliano (Poljak, 1991) y, en particular, de los regionalismos italianos traducidos al castellano, se han elegido varias fuentes documentales como el Vocabolario Siciliano (VS, 1977-2002), los principales diccionarios italianos (Battaglia, 1962-2002, Treccani, 1986-94, De Felice - Duro, 1993, Devoto Oli, 1995, De Mauro, 1999-2000 y Zingarelli, 2003), los diccionarios bilinguies (Ambruzzi, 1996 y Tam, 1997), los diccionarios monolingües (Moliner, 1994 y DRAE, 1998) y la gramática descriptiva del español dirigida por Ignacio Bosque (Bosque - Demonte, 1999). Estos instrumentos científicos han sido indispensables como fuentes externas, para integrar la fuente interna, es decir, el conocimiento personal del dialecto del área agrigentina, la zona en la que se integra la realidad dialectal de Sciascia. 
sido la de mantener el término original con nota del traductor, porque omertà es un término ampliamente conocido ${ }^{17}$. En español términos como mafia, vendetta, omertà resultan casi jergales, y al traducirse empobrecen su capacidad evocativa.

Hay casos no justificados e inexplicables como los que atañen a los nombres propios italianos que han mantenido la forma original: Francesca, Maria Grazia, Paola, Tommaso, con dos únicas excepciones, las del nombre del protagonista, Candido, con el acento proparoxítono del español, Cándido, y el de la camarera Concetta traducido por Conchita.

Tampoco aparece justificada en el plano morfosintáctico la diversidad de soluciones que propone la traductora respecto a la traducción de las construcciones hipotácticas y paratácticas con y sin subordinante, a las dislocaciones a la derecha y a la izquierda, y a los adverbios en -mente que impiden que el lector pueda percibir los rasgos estilísticos del autor.

Hay otros casos en los que la traductora no ha tenido posibilidad de elección, como sucede en las construcciones con verba dicendi, en las que el español prefiere el indicativo al subjuntivo, o en las construcciones con gerundio donde se evita su uso por varias razones: es proclive a usos agramaticales desde el punto de vista normativo, introduce pesantez de constituyentes de regusto arcaico, y es menos preciso en la ubicación temporal-aspectual.

Finalmente, la falta de unidad se presenta cuando la traductora no atiende a las diferentes intenciones estilísticas y pragmáticas con las que el autor caracteriza a los personajes, como es el caso de las palabras malsonantes. En el ejemplo de mascalzone se opta en la traducción por cuatro términos diferentes, bandido, sinvergüenza, cabrón y picaro, sin percatarse de que la repetición es un rasgo caracterizador del personaje y de su rango social ${ }^{18}$.

${ }^{17}$ En español, el término omertà, si bien no se halla registrado en los diccionarios, sí se documenta en textos periodísticos relativos a la mafia (Cfr. El País, 20-07-1980 y 01-10-1984). En ellos, omertà aparece en ocasiones explicado mediante el sintagma la ley del silencio; en otros casos, carece de explicación, dando por supuesto el conocimiento del significado por parte del lector. Llama la atención que Poljak (1991) recurra a la expresión hermandad de la mafia, que ni siquiera está registrada en los diccionarios, para traducir este término tan difundido en determinados contextos. El ejemplo aquí reseñado, así como los que aparecen en las notas siguientes, está dispuesto simétricamente en duplicado, seguido por un número entre paréntesis que indica la página de referencia. Con a se hace referencia a la edición italiana original de Candido (Sciascia, 1977). Con b se hace referencia a la edición española (Poljak, 1991). En el interior de la cita, en cada caso, aparece en cursiva el término objeto de análisis:

a. Lo insultò in nome della lealtà, dell'omertà, dell'amore alla famiglia che lui rappresentava e che Candido non conosceva né, da verme qual era, avrebbe mai conosciuto [...] (45).

b. Lo insultó en nombre de la lealtad, de la hermandad de la mafia, del amor a la familia que el abuelo representaba y que Cándido no conocía ni conocería, porque era un gusano [...] (77).

${ }^{18}$ Como ponen en evidencia los ejemplos, en los que aparece el término mascalzone en estilo directo o reproducido, el uso recurrente de esta palabra constituye un rasgo del idiolecto del general. Una traducción tan heterogénea provoca la pérdida de la función caracterizadora del término:

1a. - Per chi hai votato, per chi ti ha fatto votare quel mascalzone? (45).

1b. - ¿Por quiến has votado, por quién te ha hecho votar ese sinvergüenza? (77).

2a. Nella sua rabbia, il generale si lascio ancora andare - Parlo come mi pare; e tu - rivolgendosi a Candido - da oggi in poi non vai più da quel mascalzone - (46). 
Lo mismo sucede en el caso del verbo incalzare / acosar al que huye. El verbo hacer, utilizado en la traducción ${ }^{19}$, se aleja notablemente del significado original del enunciado, cuyo sentido no es simplemente hacer preguntas, sino el modo acuciante con el que se formulan. Por lo tanto se hubiera debido utilizar el verbo acosar, cuyo significado se acerca más al verbo incalzare.

Otro caso en el que se pierde la fidelidad al texto original es en la traducción yo me desentiendo ${ }^{20}$ en lugar de yo me lavo las manos, expresión gramaticalizada de origen bíblico. Este hecho manifiesta no sólo la pérdida de fidelidad al texto sino probablemente un problema de falta de cultura.

Aparecen también ciertas inexactitudes léxicas que alteran el original: uno de los errores más notable se encuentra en la interpretación de gebbia ${ }^{21}$. El texto original utiliza dialectalismos recurrentes en contextos descriptivo-narrativos, pertenecientes a un subcódigo particular y específico ausentes en los diccionarios de la lengua italiana. Así ocurre con gebbia que indica en español una 'tina grande de albañilería donde se conserva el agua para la irrigación'. El sustantivo acequia usado por la traductora no tiene el mismo sentido del regionalismo italiano gebbia, ya que gebbia corresponde a la palabra aljibe término distinto a acequia 'zanja para conducir el agua'.

Es oportuno resaltar el hecho de que, a veces, en un contexto ambiguo la incomprensión del texto por parte de la traductora ha ocasionado la aparición de desaciertos, no sólo en la traducción de regionalismos sino también en la de términos no regionales, propios de la lengua italiana.

En un contexto no ambiguo, el texto vuelve a ser fácilmente comprensible y dicha traductora que se presupone conoce las respectivas gramáticas de las lenguas en cuestión, tendría que haber evitado los desatinos no justificables que han modificado el sentido del original. Son inolvidables los casos de: juzgar por manejar, al pues bien, que en español no significa nada, por al caso concreto, llevar en lugar de traer, bella de noche por dondiego de noche, el no idiomático en español gloria mía en lugar de las comúnmente utilizadas vida mía, cielo mío equivalentes en el valor afectivo, entre muchos más.

2b. En medio de su rabia, el general todavía siguió desmandándose: - Hablo como mejor me parece; y tú - se volvió hacia Cándido - , desde hoy no irás más a casa de ese grandísimo cabrón (79).

3a. [...] chiamò mascalzone Candido [...] (77).

3b. [...] Llamó bandido a Cándido [...] (125).

4a. [...] rimorsi nei riguardi del generale: per non avergli dato il voto e per non avergli prestato fede quando aveva detto che l'allora arciprete era un mascalzone già, anche prima di essere degradato e di spretarsi; (79).

4b. [...] remordimientos ante el general: por no haberle dado su voto y por no haber prestado ódos cuando le había dicho que el que por entonces aún era arcipreste era un pícaro ya, aun antes de haber sido degradado y de colgar los hábitos; (127-28).

${ }^{19}$ a.Pero eso no bastaba a don Antonio, que hizo preguntas (119).

b. Ma a don Antonio non bastava, incalzò domande (73).

${ }^{20} \mathrm{a}$. Ma, caro amico, di questi sordidi casi io sempre me ne lavo le mani... (112).

b. Ah, mi querido amigo, de estos casos sórdidos yo me desentiendo siempre... (176).

${ }^{21}$ a. Comprò dei trattori, che imparò a manovrare; fece costruire condotti e gebbie per sfruttare l'acqua che prima si disperdeva; (84).

b. Compró unos tractores, que aprendió a manejar; hizo construir canales y acequias para aprovechar el agua que antes se perdía; (135). 


\section{CONCLUSIONES}

Como conclusión del análisis efectuado se puede señalar, sin volver sobre lo que ya se ha dicho sobre las modalidades de la dialectalidad, la variedad y la estratificación de la prosa, que Leonardo Sciascia mezcla sabiamente las formas más altas y refinadas de la tradición escrita con las más expresivas del lenguaje hablado.

Junto a algunos matices específicamente literarios que contienen restos típicamente idiolectales, e incluso elementos de naturaleza exógena, las elecciones del autor se situan en un nivel medio-informal del italiano escrito, que reproduce el habla dialectal, en interferencia negativa y positiva con el dialecto. La interferencia negativa deja traslucir no sólo el origen siciliano y en general la procedencia meridional, sino también las variedades subregionales que se dan dentro de Sicilia. Las formas que resultan de la interferencia positiva, aun sin ser comunes a otras variedades del italiano escrito, comportan siempre la presencia encubierta de los correspondientes dialectalismos. La lengua de Leonardo Sciascia tiende, a través de la mediación del dialẹcto, a reducir la distancia plurisecular establecida entre el código literario y el hablado del italiano, acelerando el proceso de unificación entre la lengua literaria y la lengua hablada. A esta aproximaciión han contribuido autores como Pirandello, Saba o Moravia, cuyas aportaciones han sido ampliamente reconocidas por el escritor siciliano, aunque afirme - contradictoriamente -que el flujo de los dialectos en la lengua se ha detenido. Así el estilo de Sciascia se caracteriza por la construcción compleja con profusión de oraciones incrustadas, incisos modalizadores actitudinales, aposiciones, que dotan a la secuencia de escasa progresión informativa y ponen de relieve cierto barroquismo y gusto cultista. La opción estilística de reflejar el hecho dialectal y el registro coloquial aparecen respaldados ideológicamente por el marxismo de Sciascia.

\section{Bibliografía}

Ambroise, C. (1996): Sciascia e la teologia, en SCIUTI Russi (1996: 89-136).

Ambroise, C. (ed.) (1987): Leonardo Sciascia. Opere 1956-1971, Bompiani, Milano.

- (1989): Leonardo Sciascia. Opere 1971-1983, Bompiani, Milano.

- (1991): Leonardo Sciascia. Opere 1984-1989, Bompiani, Milano.

AMBRUZZI, L. (1996): Nuovo dizionario spagnolo-italiano e italiano-spagnolo, 2 vols., VII ed., XXII reim., Paravia, Torino.

BALDI, A. (1978): Il "Candido» di Sciascia, ovvero utopia e eresia, en «Misure critiche», VIII, 28-29, luglio - dicembre, pp. 161-68.

Bandinelli, A. (1998): Candide in Parlamento, en Cincotta - CARAPEZza (1998: 187-96). Bartolotta, S. (1999-2000): Perfil de historia lingüística de Sicilia en la edad aragonesa y castellana, memoria de investigación, UNED, Madrid.

- (2002-03): La versión española de "Candido» de Leonardo Sciascia: estudio lingüístico de los regionalismos, tesis doctoral, UNED, Madrid.

BATTAGLIA, S. (1962-2002): Grande dizionario della lingua italiana, 21 vols., Utet, Torino. BERNARDINI NAPOLETANO, F. (1996): Leonardo Sciascia, dalla parte di Voltaire, en SIMONETTA (1996: 65-74). 
BernaRdini NAPOlETANo, F. (ed.) (1993): Leonardo Sciascia. La mitografia della ragione, Lithos, Roma.

Bosque, I.; Demonte, V. (eds.) (1999): Gramática Descriptiva de la Lengua Española, vol. I Sintaxis básica de las clases de palabras, pp. 1-1517, vol. II Las construcciones sintácticas fundamentales. Relaciones temporales, aspectuales y modales, pp. 1518-3503, vol. III Entre la oración y el discurso. Morfología, pp. 3504-5351, Espasa Calpe, Madrid.

BUCCA, A. (1999): «Fuoco alle polveri». Lingua e traduzione in Sciascia, en «Segno», Leonardo Sciascia. Un uomo che non si stancò di ragionare, n. 209, Palermo, pp. 141-50.

Chevello, C. (1989): Verità Letteratura e Storie. Un'altra nota su Leonardo Sciascia, en «Segno», n. 110, dicembre, Palermo, pp. 39-46.

CincotTA, R.; CARAPEZZA, M. (eds.) (1998): Il piacere di vivere. Leonardo Sciascia e il dilettantismo, «Quaderni di Leonardo Sciascia», n. 3, La Vita Felice, Milano.

ColetTI, V. (1993): Storia dell'italiano letterario dalle origini al Novecento, Einaudi, Torino.

COTTONE, G. (1980): $l l$ «Candido» di Leonardo Sciascia tra ironia e umorismo, en «Annali del Liceo Classico «Giuseppe Garibaldi» di Palermo», 14-16 (1977-79), pp. 128-31.

D'ANNA, R. (1996): Due note in margine a Candido in Sicilia, en SIMONETTA (1996: 75-84).

De Felice, E.; Duro, A. (1993): Vocabolario italiano, SEI - Palumbo, Torino - Palermo.

DEIDIER, R. (1993): L'allegoria della ragione. "Candido" tra Sciascia e Voltaire, en BERNARDINI NAPOLETANO (1993: 35-68).

DELlA MONICA, W. (1981): I dialetti e l'Italia. Inchiesta fra scrittori poeti sociologi specialisti, Pan, Milano.

De Mauro De Mauro, T. et alil (1999-2000): Grande dizionario italiano dell'uso, 6 vols., Paravia, Torino.

Devoto, G.; Olı, G.C. (1995): Il dizionario della lingua italiana, Le Monnier, Firenze.

DRAE (1998): Diccionario de la lengua de la Real Academia Española, 2 vols., XXI ed., Madrid.

Ferroni, G. (1991): Storia della letteratura italiana, 4 vols., vol. IV Il Novecento, Einaudi, Milano.

LUPERINI, R. (1978): Sciascia, la naturalità e il razionalismo, en «Belfagor», XXXIII, fasc. I, n. 1, gennaio, pp. 105-07.

Llovet, J. (ed.) (1996): Lecciones de Literatura Universal. Siglos XII a XX, con prólogo de M. de Riquer y epílogo de J. M. Valverde, Institut d'Humanitats de Barcelona, Ajuntament de Girona, Ediciones Cátedra, Madrid.

MOLINER, M. (1994): Diccionario de uso del Español, 2 vols., I ed., XIX reim., Gredos, Madrid.

MONFORTE, E. (2001): I teatri di Leonardo Sciascia, Salvatore Sciascia Editore, Caltanissetta - Roma.

MounIN, G. (1965): Teoria e storia della traduzione, Einaudi, Torino.

NAVARRo SALAZAR, M. T. (1999): Llevaba a España en mi corazón, en «ABC Cultural», 20 novembre 1999.

- (2001): Leggere Sciascia in chiave ispanica, en TEDESCo (2001: 21-43). 
Padovani, M. (ed.) (1979): La Sicile comme métaphore. Conversations en italien avec Marcelle Padovani, Editions Stock, Paris (ed. it. La Sicilia come metafora, Mondadori, Milano).

Pecoraro, Z.; Scrivano, E. (eds.) (1991): Omaggio a Leonardo Sciascia, Atti del Convegno, Agrigento, 6-8 aprile 1990, Provincia di Agrigento.

Pellegrini, S. (1970): Di Pasolini e di Gadda, en «Bollettino del Centro di Studi filologici e linguistici siciliani», 11, dedicato a «Lingua parlata e lingua scritta», Convegno di studi 1967, Palermo, pp. 366-71.

Poljak, A. (1991): Cándido o Un sueño siciliano, traducción de Ana Poljak, Tusquets Editores, Barcelona.

RAMONEDA, J. (1996): Voltaire, en Llovet (1996: 375-85).

RoHLFS, G. (1949-54): Historische Grammatik der italienischen Sprache und ihrer Mundarten, vol. I Lautlehre, vol. II Formenlehre und Syntax, vol. III Syntax und Wortbildung, Francke, Bern (tr. it. Grammatica storica della lingua italiana e dei suoi dialetti, vol. I Fonetica, vol. II Morfologia, vol. III Sintassi e formazione delle parole, Einaudi, Torino, 1966-69).

SANTANGElo, G. S. (1996): Leonardo e la statua. Note di Sciascia e l'illuminismo, en SIMONETTA (1996: 85-120).

SCARAno, E. (1993): Sciascia, Candido, Candide, en «Italianistica», XXII, nn. 1-3, gennaio-dicembre, pp. 297-316.

SCIASCIA, L. (1953): Pirandello e il pirandellismo, Salvatore Sciascia Editore, Caltanissetta - Roma.

- (1956): Le parrocchie di Regalpetra, Laterza, Roma - Bari.

- (1958): Gli zii di Sicilia, Einaudi, Torino.

- (1961): Pirandello e la Sicilia, Salvatore Sciascia Editore, Caltanissetta - Roma.

- (1966): I mafiosi, Piccolo di Milano (reim. en «Il Dramma», nn. 11-12, nov.-dic. 1972, pp. 48-72 y en SCIASCIA, 1976).

- (1970): La corda pazza. Scrittori e cose della Sicilia, Einaudi, Torino.

- (1974): Todo modo, Einaudi, Torino.

- (1976): L'onorevole. Recitazione della controversia liparitana dedicata ad A.D. I mafiosi, Einaudi, Torino.

- (1977): Candido ovvero un sogno fatto in Sicilia, Einaudi, Torino.

- (1984): Occhio di capra, Einaudi, Torino.

SCIUTI RussI, V. (ed.) (1996): Il «tenace concetto»: Leonardo Sciascia, Diego La Mattina e l'Inquisizione in Sicilia, Atti del Convegno di Studi, Racalmuto, 20-21 novembre 1994, Salvatore Sciascia Editore, Caltanissetta - Roma.

SGROI, S. C. (1984): Leonardo Sciascia fra dialetto e italo-americano, en «Studi Italiani di Linguistica Teorica ed Applicata», XIII, 2-3, pp. 411-45.

- (1987): Le parole di Sciascia, en «Teatro Stabile di Catania», La teatralità nelle opere di Leonardo Sciascia, Assessorato Regionale ai Beni Culturali, Catania, pp. 19-49.

- (1990a): Per la lingua di Pirandello e Sciascia, Salvatore Sciascia Editore, Caltanissetta - Roma.

- (1990b): Per una linguistica siciliana tra storia e struttura, Sicania, Messina.

SiCILIANo, E. (1977): Il libro scritto da Leonardo Sciascia pensando a Voltaire. Candido in Sicilia, en «Corriere della Sera», 28 dicembre 1977. 
SimONETTA, M. (ed.) (1996): Non faccio niente senza gioia. Leonardo Sciascia e la cultura francese, Atti del Seminario, Roma, 1995, «Quaderni di Leonardo Sciascia», n. 1, La Vita Felice, Milano.

TAM, L. (1997): Dizionario Spagnolo - Italiano - Diccionario Italiano - Spagnolo, Editore Ulrico Hoepli, Milano.

Tedesco, N. (ed.) (2001): Avevo la Spagna nel cuore, Atti del Convegno Internazionale, Istituto italiano per gli studi filosofici, Napoli, 15-16 ottobre 1999, «Quaderni Leonardo Sciascia», La Vita Felice, Milano.

TekavčIC, P. (1980): Grammatica storica dell'italiano, 3 vols., Il Mulino, Bologna (orig. en serbocroato 1962-67).

TreCCANI Duro, A. ET ALII (1986-94): Vocabolario della lingua italiana, vols. I, II, III*, III* y IV, Istituto della Enciclopedia Italiana fondata da G. Treccani, Roma.

VECCHIO, S. (1990): «L'italiano e il ragionare». L'idea di lingua di Leonardo Sciascia, en «Segno», XVI, n. 116, luglio-agosto, Palermo, pp. $49-57$ (reimp. con el título Sciascia e la lingua en PeCORARo - SCRIvano, 1991: 83-94).

- (1998): Un dilettante potenzialmente professionista, en CINCOTTA - CARAPEZZA (1998: 173-86).

WeinReich, U. (1953): Languages in Contact, Publication of the Linguistic Circle of New York, New York $\left(1963^{2}\right.$, Mouton, The Hague; tr. it. Lingue in contatto, con ensayos de G. Francescato, C. Grassi y L. Heilmann, Boringhieri, Torino, 1974).

ZINGARELLI (2003): Lo Zingarelli 2004. Vocabolario della lingua italiana de Nicola Zingarelli, XII ed., eds. Miro Dogliotti y Luigi Rosiello, Zanichelli, Bologna. 